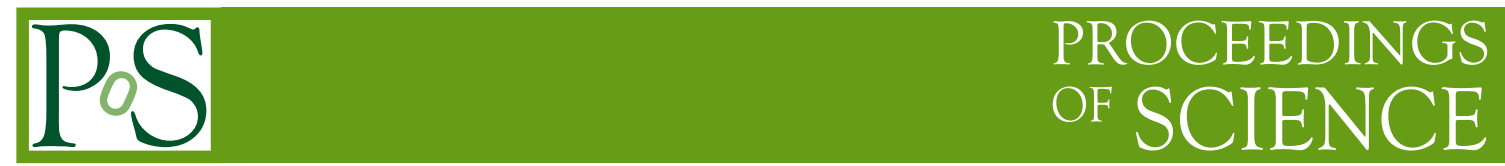

\title{
The Cosmological Standard Model
}

\section{Riotto Antonio*}

Department of Theoretical Physics and Center for Astroparticle Physics (CAP)

24 quai E. Ansermet, CH-1211 Geneva 4, Switzerland

E-mail: antonio.riotto@unige.ch

A short introduction to the Cosmological Standard Model is given, with emphasis on the predictions from inflation and the dark matter problem.

EPS-HEP 2017, European Physical Society conference on High Energy Physics 5-12 July 2017

Venice, Italy

${ }^{*}$ Speaker. 


\section{Introduction}

The incipt of the 1859 novel The Tale of Two Cities by Charles Dickens

It was the best of times, it was the worst of times, it was the age of wisdom, it was the age of foolishness, it was the epoch of belief, it was the epoch of incredulity, it was the season of Light, it was the season of Darkness, it was the spring of hope, it was the winter of despair, $\cdots$

beautifully summarises the state-of-the-art of modern cosmology. It will be soon clear why.

Any cosmological model to be successful must

- Provide a satisfactory prediction of the physics of the early Universe.

- Provide initial conditions for the origin of the cosmological perturbations, explain their evolution and the statistical properties of the cosmic structure we observe in the Universe.

- Correctly describe the dynamics of the Universe.

- Determine the cosmological parameters and provide a fundamental understanding of such quantities.

There are various tests one can perform in order to evaluate if any cosmological model reproduces the observational knowledge in our possession. The kinematical tests probe the content of the Universe and its geometry and they do so through, for example, measurements of the expansion rate, the luminosity and angular diameter distance. Ref. [1] offers a fantastic $2.4 \%$ determination of the local value of the Hubble constant $H_{0}=73.24 \pm 1.74 \mathrm{~km} \mathrm{sec}^{-1} \mathrm{Mpc}^{-1}$, see Fig. 1 .

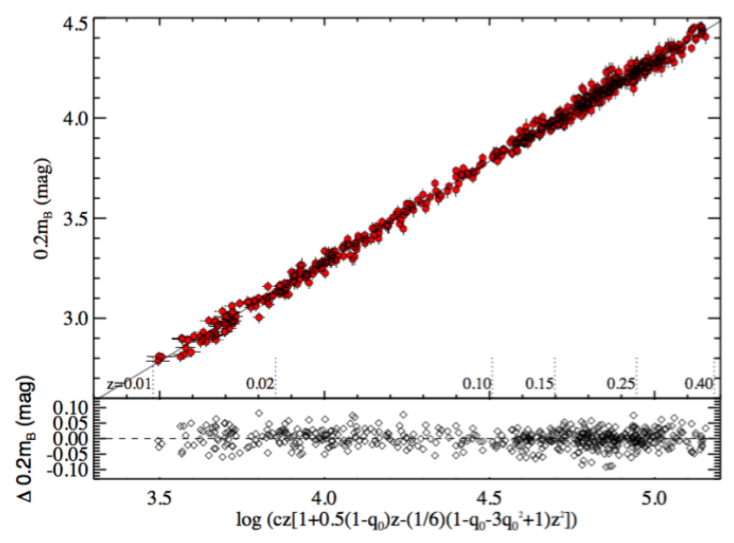

Figure 1: The Hubble diagram of more than $600 \mathrm{SNe}$ Ia at redshifts $0.01<z<0.4$. From Ref. [1].

Another essential test is the structure formation probe, which is supposed to give us information about the content of the Universe and the nature of the perturbations. It takes place through measurements of the Cosmic Microwave Background (CMB) anisotropies as well as of the Large Scale Structure (LSS). These two subjects have been addressed by the previous two speakers and we will 
not go further in detail. Suffice to say that the Cosmological Standard Model is now a synonym for the so-called $\Lambda \mathrm{CDM}$ model or concordance model. It is a few-parameter model which assumes a flat Universe and a non-vanishing cosmological constant $\Lambda$ and is able to fit a large amount of current data. The fundamental inout parameters are

1. The amount of Cold Dark Matter (CDM), $\Omega_{\mathrm{CDM}}$.

2. The amount of baryons, $\Omega_{\mathrm{b}}$.

3. The spectral index of the primordial cosmological perturbations, $n_{\zeta}$.

4. The amplitude of the primordial cosmological perturbations, $A_{\zeta}$.

5. The reionization optical depth $\tau$.

The $\Lambda \mathrm{CDM}$ has shown some tension with the recent local determination for the Hubble constant

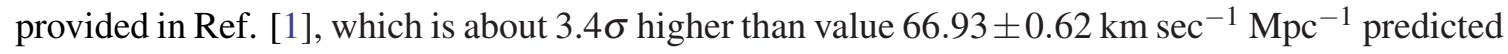
by the concordance models with three neutrinos with mass $0.06 \mathrm{eV}$ and the Planck data [2]. While this discrepancy is certainly not due to sample variance, it might well due to some systematics in both measurements. If not so, the origin of the difference might be ascribed to some fluid with an equation of state $w<-1$ or to some interaction between the dark energy and the dark matter. In any case to something that goes beyond the $\Lambda \mathrm{CDM}$ model. Only the future will tell us.

The real problem with the $\Lambda$ CDM model is what we dubbed the Fundamental Understanding Problem. The $\Lambda \mathrm{CDM}$ model delivers an almost perfect fit to the current data at various cosmological scales and epochs as long as $\Omega_{\Lambda}=0.685 \pm 0013$ and $\Omega_{\mathrm{CDM}}=0.315 \pm 0.013$. However, we do not have the slightest idea of the origin of such minuscule cosmological constant and we do not know anything about the origin of the DM. The situation is quite similar to what happens in the Standard Model of weak interactions. We know it nicely describes the interactions up to the $\mathrm{TeV}$ energy scale, but we are ignorante about the origin of the baryon asymmetry, the neutrino masses, the flavour structure, not to mention the naturalness problem affecting the Higgs sector. Nevertheless, cosmology provides a fantastic playground for HE physicists and in this talk we will touch upon (with some bias) two pillars of the Cosmological Standard Model: the initial conditions provided by inflation and DM.

\section{First pillar: inflation}

There is little doubt among most of the members of the cosmology community that the Universe went through a period of accelerated expansion [3], most likely sourced by a scalar field, dubbed the inflaton, with a very flat potential inducing a slow-roll dynamics in such a way that the energy density of the universe is driven by the potential energy of the inflaton field $\phi$

$$
H^{2}=\left(\frac{\dot{a}}{a}\right)^{2} \simeq \frac{V(\phi)}{3 M_{\mathrm{Pl}}^{2}} \Rightarrow a(t) \sim e^{H t} .
$$

One first consequence of this exponential expansion is that the local space curvature is reduced basically to zero 


$$
\Omega-1=\frac{k}{a^{2} H^{2}}=\mathscr{O}\left(e^{-2 N}\right),
$$

where $N$ is the number of $e$-folds to the end of inflation. Since $N$ is larger than 40 or so, one automatically deduces that inflation predicts the current value of $\Omega$ to be extremely close to unity. Current Planck data give $\Omega_{\Lambda}+\Omega_{\mathrm{CDM}}=0.685+=0.315=1$ with a permille error. This is a fantastic prediction of inflation being confirmed.

The second incredible gift inflation gave us is its explanation of the CMB anisotropies and the LSS we observe in the universe. The idea is the following. During the fast and accelerated inflationary expansion, small perturbations are generated at the quantum level. This is an unavoidable consequence any time the Universe expands or contracts. Indeed, at the microscopic level pairs of particles are generated and destroyed making the vacuum dynamical. In a static situation these newly-bork particles will annihilate and go back to the vacuum. However, in an exponentially expanding Universe, these particles are driven far apart and from virtual they become real particles. An external observer will see pairs of particles popping out. They can be thought as perturbations of the background.

Another important aspect of these perturbations is that their physical wavelength grows with time, $\lambda=(2 \pi / k) a$, where $k$ is the comoving momentum. This means that, even though the perturbations are originated at quantum scales, their wavelenghts are stretched to cosmological scales. In doing so, from quantum they become classical. Gravity or, better to say, the gravitational instability phenomenon due to the attractive property of the gravitational force, will do the rest by making these tiny perturbations become clumpier and clumpier.

From a more mathematical point of view one can show that any massless scalar field $\sigma(\vec{x}, t)$ is quantum-mechanically excited during inflation. Let us from its action

$$
S=\int \mathrm{d}^{4} x \frac{1}{2} \sqrt{-g}(\partial \sigma)^{2} .
$$

By splitting the scalar field into its zero-mode and the perturbations,

$$
\sigma(\vec{x}, t)=\sigma_{0}(t)+\delta \sigma(\vec{x}, t)
$$

and by introducing the conformal time $\mathrm{d} \tau=\mathrm{d} t / a(t)$, one can show that the equation of motion for the Fourier transform $u_{\vec{k}}(\tau)=a(\tau) \delta \sigma_{\vec{k}}(\tau)$ becomes [3]

$$
u_{\vec{k}}^{\prime \prime}+\left(k^{2}-\frac{a^{\prime \prime}}{a}\right) u_{\vec{k}}=0 .
$$

The corresponding power spectrum on large scales, that is for $\lambda \gg H^{-1}$ is flat

$$
\mathscr{P}_{\delta \sigma}=\frac{k^{3}}{2 \pi 2}\left|\delta \sigma_{\vec{k}}\right|^{2}=\left(\frac{H}{2 \pi}\right)^{2} k^{0} .
$$

This surprising result, the fact that the power spectrum of the perturbations of a massless scalar field does not depend on its wavelength, is in fact a direct consenquence of the so-called dS/CFT correspndance [4]. The metric during a period where the energy density of the Universe is driven by a constant vacuum energy is called de Sitter and reads 


$$
\mathrm{d} s^{2}=\frac{1}{H^{2} \tau^{2}}\left(\mathrm{~d} \tau^{2}-\mathrm{d} \vec{x}^{2}\right)
$$

This metric is clearly invariant under dilations, $\tau \rightarrow \lambda \tau$ and $\vec{x} \rightarrow \lambda \vec{x}$. Furthrmore, it is also invariant under special conformal transformations, spatial rotations and translations. At the hypersurface identified by $\tau=0$, the end of inflation, all these transformations for the group $\mathrm{CFT}_{3}$, the threedimensional group of conformal transformations. Since, for instance, under dilations the perturbations transform as $\delta \sigma_{\vec{k}} \rightarrow \lambda^{-3} \delta \sigma_{\vec{k} / \lambda}$ and the action is invariant under dilations, the power spectrum must be scale-invariant. One other way to mathematically state that is that the spectral index of the power spectrum, $\mathscr{P}_{\delta \sigma} \sim k^{n_{\delta \sigma}-1}$, must be equal to unity, $n_{\delta \sigma}=1$.

Of course, during a realistic stage of inflation, the metric may not be exactly de Sitter, otherwise inflation will never end: the inflaton must slowly rolls along a potential which is slightly tilted. Inflation therefore predicts a small breaking of scale-invariance. In the high energy physics language, the slightly broken scale invariance is associated to a pseudo Nambu-Goldstone boson representing fluctuations in the clock. Different regions expands slightly differently because of a non-vanishing comoving curvature perturbation $\zeta$

$$
\zeta \sim \frac{\delta a}{a} \sim H \delta t \sim H \frac{\delta \phi}{\dot{\phi}_{0}} .
$$

Because of this simple logic, the spectral index of $\zeta$ is expected to be different, but close to unity. The fact that Planck has set up its value around 0.96 is another astonishing prediction of inflation which has become true

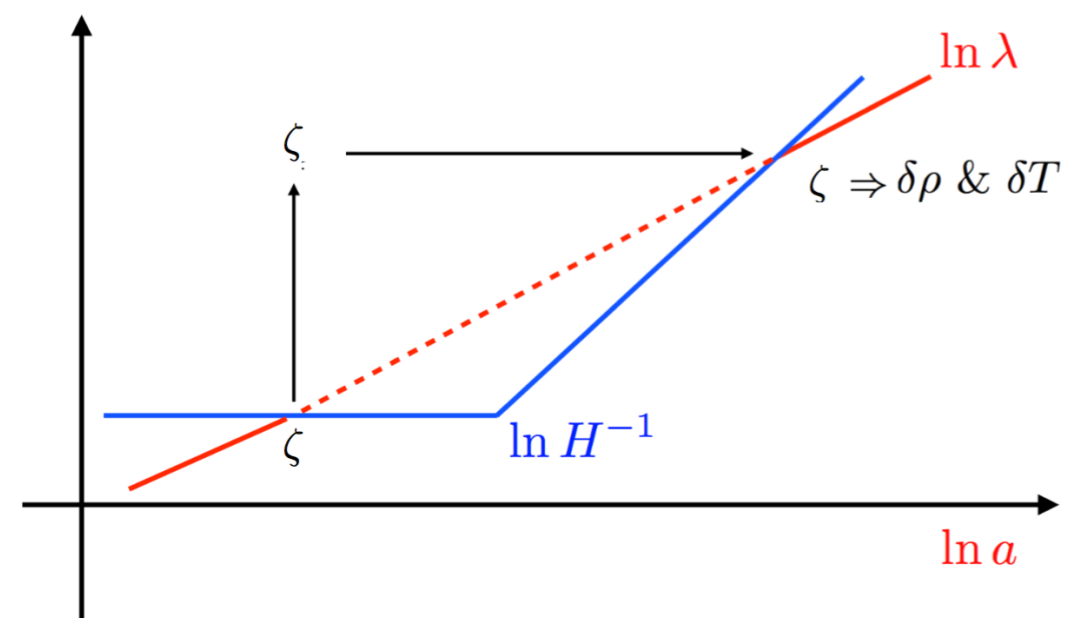

Figure 2: The evolution of the comoving curvature perturbation.

The characterization of the power spectum generated during inflation is only the first step. We are more and more convinced that the LSS we see in our Universe as well as the CMB anisotropies owe their origin to some primeval inhomogeneities generated during primordial inflation. Yet, the precise mechanism by which cosmological perturbations are generated is not established. In 
the standard single-field models of inflation where there is a universal clock, the inflaton, density perturbations may be ascribed to fluctuations of the inflaton itself when it slowly rolls down along its potential. In the curvaton mechanism [5] the final curvature perturbation $\zeta$ is produced from an initial isocurvature mode associated with the quantum fluctuations of a light scalar (other than the inflaton), the curvaton, whose energy density is negligible during inflation.

The observable which proves fundamental in providing information about the mechanism chosen by Nature to produce the structures we see today is Non-Gaussianity (NG), that is the deviation from a Gaussian statistics through the presence of higher-order connected correlation functions of the perturbations. Indeed, a possible source of NG could be primordial in origin, being specific to a particular mechanism for the generation of the cosmological perturbations. This is what makes a positive detection of NG so relevant: it might help in discriminating among competing scenarios which otherwise might be undistinguishable. Let us consider the three-point correlator in the squeezed limit, in which one wavelength is much larger than the others. In single-field models, the effect of such a constant long-wavelength mode on an $n$-point function may be reproduced as the rescaling of the coordinates

$$
\left\langle\zeta\left(\vec{x}_{1}\right) \cdots \zeta\left(\vec{x}_{n}\right)\right\rangle_{\zeta_{L}}=\left\langle\zeta\left(\vec{x}_{1}^{\prime}\right) \cdots \zeta\left(\vec{x}_{n}^{\prime}\right)\right\rangle .
$$

This argument implies that in that case the squeezed limit of the $(n+1)$-point function would be

$$
\left\langle\zeta_{\vec{q}} \zeta_{\vec{k}_{1}} \cdots \zeta_{\vec{k}_{n}}\right\rangle_{q \rightarrow 0}^{\prime}=P_{\zeta}(q)\left[3(n-1)+\sum_{a=1}^{n} \vec{k}_{a} \cdot \vec{\nabla}_{k_{a}}\right]\left\langle\zeta_{\vec{k}_{1}} \cdots \zeta_{\vec{k}_{n}}\right\rangle^{\prime},
$$

where primes indicate we have removed pi's and Dirac delta functions. For $n=2$, the relation above provides the famous Maldacena's consistency relation for the three-point correlator of the comoving curvature perturbation in the squeezed limit. It will be badly violated in curvaton-like models. Hence, the extremely relevance of an accurate measurement of the three-point-correlator.

A violation of the consistency relation (2.10) would indicate the presence of non-trivial physics during inflation. The question is then which models can violate the consistency relation and which signatures to expect from them. As already mentioned, a violation of the consistency relation could indicate the presence of additional degrees of freedom. This requires a careful study of the physics of higher-spin fields during inflation, which has attracted a lot of attention recently [6, 7]. The problem of writing down consistent equations of motion and Lagrangians for higher-spin fields goes back to the beginning of quantum field theory and is particularly difficult fo massless fields. Massless degrees of freedom with spin $s \geq 1$ are gauge fields and they come with a corresponding gauge invariance needed to decouple unphysical polarisations. The problem of writing consistent self-interactions become therefore highly constrained and complicated.

In flat space one can write down consistent gauge-invariant equations of motion for the free fields, but it seems impossible to have non trivial $S$-matrices for spins $s>2$ since the gauge invariances are accompanied with conserved charges and the conservation laws are too strong to allow non-trivial $S$-matrices. On the other hand, there are some explicit constructions of self-interacting massless HS theories away from flat spacetime when a non-vanishing cosmological constant is allowed. This is particularly interesting when thinking of the possible role of higher spin fields during inflation. These theories always contain the massless spin-2 graviton and are therefore theories of 
gravity. An important feature of these higher-spin theories in is that mathematical consistency implies that they involve an infinite tower of fields of all spins. Of course, to assess the importance of massless higher spin fields during inflation one has to deal not only with the infinite tower of degrees of freedom (which might turn out to be a bonus from the observational point of view), but also to compute the couplings of the massless HS fields to the matter (inflaton) sector.

\section{Second pillar: dark matter}

There is no dispute that the DM in cosmology and HEP has been dominated by the WIMP paradigm, i.e. that of a neutral cold particle with weak interactions whose abundance is determined by the freeze-out to be

$$
\Omega_{\mathrm{CDM}} \simeq \frac{3 \cdot 10^{-27} \mathrm{~cm}^{3} \mathrm{sec}^{-1}}{\left\langle\sigma_{\mathrm{A}} v\right\rangle} .
$$

The fact that weak interactions imply an abundance in the ballpark of what measured is called the WIMP-miracle. A strong point of the WIMP is its complementarity search chart, see Fig. 3.

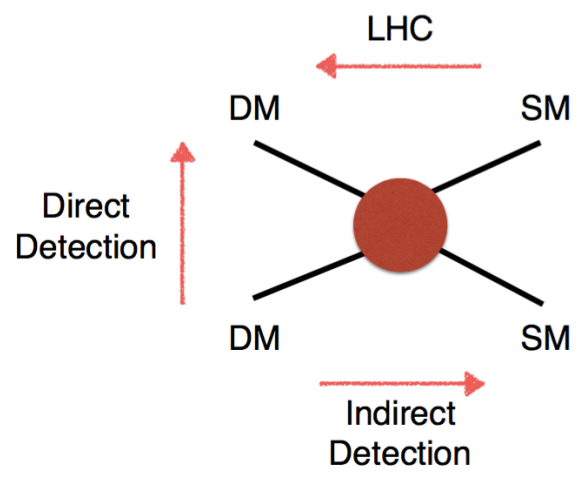

Figure 3: The WIMP complementarity search chart.

Nevertheless, in the absence of any signature, the situation is becoming similar to what our HEP fellows are into now: naturalness calls for supersymmetry, but no sign of it. WIMP's may be around the corner, but for sure it is healthy to cope with

- the moderate idea that DM might have another origin, other interactions, production mechanisms (good for HEP),

- and maybe with the extreme (and hopefully wrong idea) that it manifests itself only through gravitational effects.

One possible moderate variation away from the WIMP is the ultralight axion, i.e. a pseudo-scalar particle with mass $m_{a}$ around $10^{-22} \mathrm{eV}$, whose de Broglie length therefore is around the Kpc [8]. The beauty of this candidate is its quantum nature at the Kpc scale. Indeed, its wave-function satisfies a Gross-Pitaevskii-Poisson equation of the form 


$$
i \partial_{t} \psi=-\nabla^{2} \psi / 2 m_{a}+m_{a}\left(\Phi-g|\psi|^{2} / 8\right) \psi,
$$

where $\Phi$ is the corresponding gravitational potential. This system is in fact a Bose-Einstein condensate (in the cosmo) and the kinetic term $\nabla^{2} \psi$ offers the source for the quantum pressure at small scales. Recent constraints on the masses of these extremely light bosons from Lyman- $\alpha$ forest data seems to rule out such a possibility [9]. However, non-linearities, which are fundamental in highly populated environments have not been accounted for.

\section{Conclusions}

Cosmology offers a fantastic playground for HE physicists where they can test their theories or invent new ones to fit the observations in the cosmo. This relation is more intimate than ever.

\section{References}

[1] A. G. Riess et al., Astrophys. J. 826, no. 1, 56 (2016) doi:10.3847/0004-637X/826/1/56 [arXiv:1604.01424 [astro-ph.CO]].

[2] F. Bouchet, Cosmic Microwave Background, to be published in PoS(EPS-HEP2017)577 (this conference).

[3] D. H. Lyth and A. Riotto, Phys. Rept. 314, 1 (1999) doi:10.1016/S0370-1573(98)00128-8 [hep-ph/9807278].

[4] A. Strominger, JHEP 0110, 034 (2001) doi:10.1088/1126-6708/2001/10/034 [hep-th/0106113].

[5] K. Enqvist and M. S. Sloth, Nucl. Phys. B626, 395 (2002) [hep-ph/0109214]; D. Lyth and D. Wands, Phys. Lett. B524, 5 (2002) [hep-ph/0110002]; T. Moroi and T. Takahashi, Phys. Lett. B522, 215 (2001) [hep-ph/0110096] [Erratum-ibid. B539, 303 (2002)].

[6] N. Arkani-Hamed and J. Maldacena, arXiv:1503.08043 [hep-th].

[7] A. Kehagias and A. Riotto, JCAP 1707, no. 07, 046 (2017) doi:10.1088/1475-7516/2017/07/046 [arXiv:1705.05834 [hep-th]].

[8] L. Hui, J. P. Ostriker, S. Tremaine and E. Witten, Phys. Rev. D 95, no. 4, 043541 (2017) doi:10.1103/PhysRevD.95.043541 [arXiv:1610.08297 [astro-ph.CO]].

[9] V. Irsic, M. Viel, M. G. Haehnelt, J. S. Bolton and G. D. Becker, Phys. Rev. Lett. 119, no. 3, 031302 (2017) doi:10.1103/PhysRevLett.119.031302 [arXiv:1703.04683 [astro-ph.CO]]. 